\section{RADIO WAVE PROPAGATION} INTERNATIONAL COLLOQUIUM IN PARIS

\begin{abstract}
A COLLOQUIUM was held in Paris during
September 17-21, at which various aspects of radio wave propagation were discussed. The proceedings were organized by the French National Committee of the International Scientific Radio Union and the Société des Radioélectriciens. The time chosen was shortly after the eighth Reunion of the International Radio Consultative Committee in Warsaw, as a result of which a number of delegates from foreign countries attended who might not otherwise have been able to do so : there were in fact representatives of more than twenty countries present. The topics discussed fell broadly under the three headings of tropospheric propagation, ionospheric propagation and general theoretical problems.
\end{abstract}

\section{Tropospheric Propagation}

In view of the current widespread interest in the possible uses of ultra-high-frequency radiation for transmission to points far beyond the horizon, it was not surprising that much of the discussion in the sessions devoted to tropospheric propagation should be concerned with the physical processes involved in such transmission. Over the past few years several theories advanced to explain the phenomenon have been debated at length. For most of this time it has been held by many workers that the cause of long-distance tropospheric fields is scattering produced by fluctuations of refractive index due to turbulence in the lower atmosphere. A less widely held view is that advocated by T. J. Carroll : it is based on the normal mode theory applied to an atmosphere in which the refractive index decreases monotonically from its value at the surface of the earth to unity at some specified height, above which it is maintained at unity : the mathematical rigour of this theory has, however, been seriously questioned.

While recognizing the powerful stimulus to research and practical applications provided by the turbulence theory, some speakers at Paris were of the opinion that a new attack on the problem was becoming desirable. The various forms of the theory proposed by E. C. S. Megaw, by H. G. Booker and W. E. Gordon and by F. Villars and V. F. Weisskopf all postulate isotropic turbulence, and it is generally realized that this must be far from true. Soundings of the refractive index of the troposphere made by centimetre-wave refractometers, for example, provide evidence of marked horizontal stratification which is nearly always present in some height interval. Modifications of the turbulence theory to allow for anisotropy have been proposed, but these, it is felt, do not meet the full needs of the problem : as a consequence there is a trend towards explaining long. distance fields primarily in terms of partial reflexion and scattering from one or more layers in which there is a significant change in the gradient of refractive index with height from that occurring in neighbouring regions of the atmosphere above and below, and which may present a rough, or at times broken, surface to the incident radiation. This point of view was strongly expressed at the colloquium by L. J. Anderson. It is evident that much more work, both theoretical and experimental, remains to be done before a really satisfactory explanation of longdistance tropospheric fields is obtained.
J. B. Wiesner presented a general account of the principles underlying the design of troposphericscatter links for communication purposes. He directed attention to the fact that the propagation mechanism is not the only factor determining the choice of the optimum frequency for a given link; that is, within the range $400-5,000$ Mc./s., which is generally regarded as being the band in which the most useful applications of tropospheric scattering can be made. Consideration must also be given to the power available for transmission, the sensitivity of receivers, and the feasibility of building the size of aerial which may be required; for if wide-band information is to be transmitted, highly directive aerials are essential. It thus appears that, for links $300-400 \mathrm{~km}$. in length, the optimum frequency is somewhere in the region of $1,000 \mathrm{Mc} / \mathrm{s}$. ; but there is an advantage in using lower frequencies, of the order of 400 $500 \mathrm{Mc} . / \mathrm{s}$. , for appreciably longer links. The economics of the problem is also of considerable importance, and this aspect was discussed by P. Clavier.

In a description of work carried out at the Lincoln Laboratory of the Massachusetts Institute of Technology, J. H. Chisholm gave details of investigations of the angular distribution of tropospherically scattered radiation, and went on to consider the communication capacity of scatter links; he emphasized the importance of multipath propagation on this capacity and the use of highly directive serials for minimizing such effects. Other work at the Lincoln Laboratory, dealing with the practical aspects of the design of long-range communication systems, was described by W. E. Morrow : the improvement in performance to be obtained by the use of spacediversity reception on scatter links was pointed out, and a demonstration was given showing the advantages of single-sideband amplitude modulation over frequency modulation for the reception of weak signals.

Much effort has been devoted at the U.S. National Bureau of Standards to the study of the characteristics of the troposphere by radio means ; and various features of this work were outlined by J. W. Herbstreit. A great quantity of information has been collected concerning the variations of field strength with time and distance from the transmitter at frequencies in the range 100-1,000 Mc./s.; and this has boen used to derive a model (statistical in nature) of conditions in the troposphere on the basis of the theory of turbulent scattering. Discussion on this topic at the colloquium was very lively, and the opinion was expressed by a number of speakers that it was perhaps unwise to apply the model for conditions-whether of climate or frequency-different from those for which the model had been derived. Other investigations at the National Bureau of Standards, described by B. R. Bean, have been concerned with the relationship between climatology and the value of the refractive index at the surface of the ground, which is correlated with transmission loss. The same speaker also gave an account of gaseous absorption of microwaves in the atmosphere in relation to tropospheric scatter systems.

The centimetre-wave refractometer has been used extensively in the past few years, particularly in the United States, for the investigation of refractive index variations in the troposphere. The results of such investigations were presented by C. M. Crain, who discussed the manner in which the refractive index varied in and around various types of cloud, 
fronts and other atmospheric disturbances. The application of measurements made by means of refractometers to studies of radio wave propagation were also considered. Correlation of a more general nature between propagation characteristics and meteorological data obtained from routine radio-sonde observations was the subject of a paper by F. du Castel.

Other papers in the tropospheric propagation section of the colloquium were concerned with: the comparison of the amplitude and phase fluctuations associated with scatter transmissions $(\mathrm{H}$. Bremmer), propagation experiments at short distances (P. Chavance), the effect of rapidly changing airmasses on tropospheric propagation (P. Misme), and optimum aerial apertures for scatter links (W. C. Hoffman).

\section{Ionospheric Propagation}

In the ionospheric section, the basic theory of reflexion of radio waves from the ionosphere was dealt with by P. Poincelot, and also by J. R. Wait, who considered mainly the problem of the propagation of atmospherics at oblique incidence and the interpretation of their waveforms. D. Lepechinsky made a contribution on the practical application of the Appleton-Hartree magneto-ionic theory: he discussed the importance of the influence of the Earth's magnetic field and the electron-collision frequency on the form of $h^{\prime} f$ records.

The general problem of oblique-incidence propagation was the subject of much discussion, and W. Dieminger described the results obtained with variable-frequency pulse transmissions over a path of $1,300 \mathrm{~km}$. Agreement between observations over this pauh and the results which would have been expected from data obtrined by ionospheric soundings at vertical incidence was good except when the oblique $p^{\prime} f$ traces showed an unexplained extension of the normal 'nose'. Measurements of short-wave field strengths and atmospheric noise made on ships sailing from Denmark to Greenland were described by C. Ungstrup ; these experiments give direct confirmation of the abnormally high absorption of radio waves which occurs over an arc roughly coincident with the auroral zone. B. Beckmann directed attention to the use of commercial transmitters for making routine 'back-scatter' measurements which can help in the assessment of propagation conditions. Detailed studies of the timing signals from standard-frequency stations in the high-frequency band wave were described by J. Bouchard, who has used these signals to investigate modes of propagation; and also by Mme. Stoyko, who made use of them in the derivation of an empirical expression for the time of propagation as a function of distance which is correct to 0.1 millisecond.

A survey of the various problems which are encountered in the preparation of ionospheric forecasts for operational use was given by $K$. Rawer. The particular problem of forecasting verticalincidence critical frequencies was discussed by C. M. Minnis, who proposed a monthly ionospheric index of solar activity which would reduce errors in forecasting to 60 per cent of the error incurred using an index based on sunspot numbers. In a contribution on the difficult question of forecasting the occurrence of ionospheric storms, P. Simon described how a distinction could be made between active and nonactive sunspots by observations of the characteristics of the emitted radio noise.
The nature of the ionospheric mechanism by which very high frequency signals may be transmitted over distances of 1,000-2,000 km. was considered by J. A. Saxton. It seems likely that such signals are predominantly propagated by reflexion from trails of meteoric ionization during the night, but that during the day there is also an important contribution which depends upon fluctuations of electron density produced by turbulence in the lower $E$-region. The part played by meteoric ionization in radio wave propaga. tion was also discussed by V. R. Eshleman, who pointed out that the factors which control the duration of a radio echo from a meteor trail include the wave-length, the electron line density in the trail, the rate of diffusion of the trail, and the geometry of the transmission path. As the path-length is increased, the average echo duration first increases to a maximum and then decreases.

There were a number of papers dealing with the propagation of very low frequency radiation including atmospherics. One of these, by F. Horner, was concerned with the possible dependence of the ionospheric transmission of very long waves on the direction of the path; and it appears probable that the polarization of the received signal does in fact depend on the horizontal direction of the path relative to the Earth's magnetic field. Papers were introduced by H. E. Dinger and R. Rivault on 'whistling atmospherics' - audio-frequency electromagnetic disturbances which are believed to have been propagated through the outer regions of the ionosphere-and discussions on this subject concentrated on the more complex and unusual types for which no theoretical explanations have as yet been advanced. F. W. Chapman also described work on audio-frequency radio waves, in the range $40 \mathrm{c} . / \mathrm{s}$. to $16 \mathrm{kc} . / \mathrm{s}$., and showed how, from an examination of the way the waveform and spectrum vary with distance of propagation, information may be obtained on the attenuation and velocity of these waves. E. T. Pierce showed that the waveforms of atmospherics received in England from sources in south-easterly directions are different from those originating at similar distances to the south-west.

Although the colloquium was mainly concerned with wave propagation, several speakers discussed problems of ionospheric physics. W. Pfister referred to recent rocket experiments which may be interpreted as confirming the existence of an irregular structure superimposed on the normal horizontal stratification in the $E$-region. A new graphical method of determining the vertical electron density distribution was outlined by W. Becker ; this method allows for the Earth's magnetic field, and is designed primarily to deal with parabolic, cosine and Epstein distributions. $E$-region wind measurements made by E. Harnischmacher indicate a mean day-time velocity of 80 metres per second, and a diurnal clockwise rotation in direction of 30 degrees per hour. Results of work on the self-demodulation of radio waves on frequencies near the gyro-frequency were presented by M. Cutolo ; and L. Goldstein described laboratory experiments on the simulation of ionospheric interaction phenomena under controlled conditions in discharge tubes.

\section{General Theoretical Problems}

Whether or not the Sommerfeld surface wave actually exists has been a topic for argument for many years, and it appears from the discussions at 
Paris, which were stimulated by papers by A. Banos and G. Goubau, that no completely satisfactory answer to the problem has yet been reached. Also, in the general theoretical section of the colloquium there was extensive consideration of diffraction problems, a leading part being played by V. A. Fock, who presented a comprehensivo paper dealing with the application of complex integrals to these problems.

A treatment of the scattering of high-frequency radiation by dielectric spheres was given by $D$. S. Saxon and $z$. Sekera. It would seem that, while satisfactory theories exist for scattering processes when the wave-length is short compared with the scale of the inhomogeneities of the scattoring medium, they are not valid at longer wavo-lengths.

A matter of considerable importance in propagation studies is the reflexion of radio weves at rough surfaces. Various aspects of this subject were discussed by W. S. Ament, who gave quantitative results for surfaces conforming to certain simple models; and also by V. Twersky, who presented a generalized theory - though here again only simplified models are amenable to numerical treatment.

Current interest in long-wave transmission has been stimulated by recent theoretical work on the problem, notably that by K. G. Budden in which the space between the Earth and the ionosphere is treated as a wave-guide. It is hoped that now experimental work will help to resolve some of the difficulties in the theory which still exist. In this connexion the study of atmospherics is important, and it was interesting to hear a report in Paris by $J$. Aaroris that measurements have now been made down to frequencies as low as 0.5 cycles per second, to supplement those which have been made for a number of years at higher frequencies.

It is proposed that the papers presented at the colloquium shall be published in a special number of L'Onde Electrique which will appear in the spring of 1957 .

\section{PHYSIOLOGY OF THE PRE-WEANING PERIOD}

\begin{abstract}
A CONFERENCE, in commemoration of $\mathrm{E}$. Babák, was held by the Czechoslovak Academy of Sciences at Liblice, near Prague, during November 14-17, 1956. The subject of the conference was "Problems of the Physiology of the Pre-weaning Period in Man and other Mammals". Countries represented included the German Democratic Republic, Great Britain, Hungary, Rumania, the United States, U.S.S.R. and Yugoslavia.

A central topic was the development of thermoregulation, on which extensive resoarches are being carried out in Prague, at the Institute of Physiology of the Academy of Sciences and at the Charles University. P. Hahn, J. Křeček, J. Křečkova and J. Martínek (Prague) contributed papers on the development of thermo-regulatory mechanisms in the rat, and on adaptation to cold and warm environments in infant rats. Othor pepers in this field included those of S. A. Barnett and B. M. Manly (Glasgow) on the effects of low environmental temperatures on young mice; K. W. Cross (London) on the reaction of the human new-born to lack of oxygen; A. Holub and others (Brno) on the development of thermorcgulation in piglets; and A. McLaren and D. Michie
\end{abstract}

(London) on the effects of low and high temperatures on growth and variability in mice.

Other aspects of homeostasis were dealt with by J. Cort (Prague), who described work on the renal tubular excretion of acids in the human new-born; and Gertrude Falk (Washington), who discussed endocrine factors in the post-natal development of water diuresis. In addition, M. Hašek (Prague) gavo an account of his important work on the adaptation of homeotherms to foreign antigens during early ontogeny.

Another major theme wes the development of the nervous system. P. K. Anochin (Moscow) described an extensive series of researches on the relationship between functional maturation in the nervous system and the appearance of innate responses. I. A. Arshavsky (Moscow) gave a paper on the nervous system in the development of gastro-intestinal and circulatory function. $K$. Capek and J. Jelinek described experiments on the external stimuli requirod to establish reflex micturition in puppies. $P$. Jilek and others discussed the development of normal brain function. J. Lát described researches in which individual variation in food choice in rats was found to be related to metabolic differences. J. L. Malcolm (London) reported on the pattern of response of motor neurons of the spinal cord in kittens, and the relationship between anatomical and functional development. A. D. Slonim (Leningrad) described a study of innate alimentary reflexes during ontogeny.

Visits were made to research institutes and medical centres in Prague. It was provisionally decided to hold a similar conference, again in Prague, in three or four years time.

S. A. Barnett

\section{FATIGUE IN METALS}

$\mathbf{N}$ the hundred years since the term 'fatigue' was first introduced to define a certain type of unexplained service failure, a great deal of empirical knowledge has been accumulated, but there is still much to learn regarding the fundamentals of the processes involved. The Institution of Metallurgists has published a series of five papers*, presented as lectures during 1955, which together summarize, possibly better than has been done before, what is known and what is not known about this obscure phenomenon.

Dr. J. Holden deals with the fundamental considerations so far as they are known in a condensed but clear manner. It has been realized for a long time that fatigue depends greatly on the nature of the surface of the part under consideration, and Mr. G. Forrest is concerned to summarize the effect of notches and surface finishes, such, for excmple, as nitriding, shot-blasting, decarburization, etc., on the resistance to fatigue, and concludes that "the only general conclusion which can be reached is that the behaviour of materials with respect to notches and surface finishes is so complicated and related to so many variables that we are still a long way from being able to estimate the strength of complicated engineering components from fatigue tests on normal laboratory specimens".

* The Fatigue of Metals : Lectures delivered at the Institution's Refresher Course 1955. Pp. 148+16 tables. (London: Institution $\begin{array}{ll}\text { Refresher Course 1955. Pp. } & \text { Pp. } \\ \text { of Metallurgists, 1956.) } 25 s .\end{array}$ 